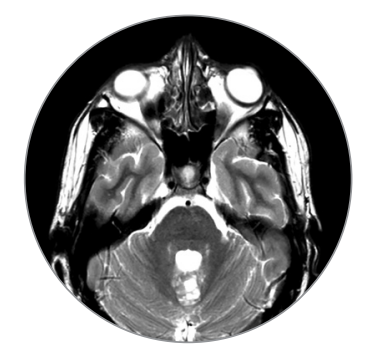

\begin{tabular}{l}
\hline Palabras clave (DeCS) \\
\hline $\begin{array}{l}\text { Imagen por resonancia } \\
\text { magnética } \\
\text { Neoplasias encefálicas } \\
\text { Cerebelo }\end{array}$ \\
\hline Key words (MeSH) \\
\hline $\begin{array}{l}\text { Magnetic resonance } \\
\text { imaging } \\
\text { Brain neoplasm } \\
\text { Cerebellum }\end{array}$ \\
\hline
\end{tabular}

'Residente de Radiología, Universidad Pontificia Bolivariana. Medellín, Colombia.

${ }^{2}$ Neurorradiólogo, CediMed, Universidad de Antioquia. Medellín, Colombia.

CediMed. Medellín, Colombia.

\title{
Lesión multinodular y vacuolar de fosa posterior. Presentación de caso en Colombia
}

\author{
Multinodular and Vacuolating Posterior Fossa Lesions of Unknown \\ Significance. Case Report in Colombia
}

\author{
Valentina Múnera \\ Sergio Vargas ${ }^{2}$ \\ Diego Alberto Herrera Jaramillo²
}

\section{Resumen}

Existen múltiples lesiones quísticas que se pueden encontrar en el encéfalo y en la fosa posterior. Entre estas, una nueva entidad denominada MV PLUS (por su sigla en inglés de multinodular and vacuolating posterior fossa lesions of unknown significance) tiene características imagenológicas similares al tumor multinodular vacuolante, pero como lo indican su sigla en inglés, está localizado en la fosa posterior. Estos tumores se definen como un conjunto de lesiones subcorticales nodulares pequeñas, de alta señal en secuencias con informaciónT2-FLAIR, con o sin realce al administrar medio de contraste. Pueden diferenciarse de otras entidades quísticas porque son lesiones agrupadas, sin cambios inflamatorios reactivos, presentes en una corteza de apariencia normal y sin evolución en el seguimiento. Esto último hace que se consideren lesiones benignas, no agresivas. Se presenta el caso de un paciente de 21 años de edad, a quien de manera incidental se le encontró en la región central del vermis una lesión de aspecto multiquístico, sin cambios durante el seguimiento en los últimos 3 años, con diagnóstico probable de MV PLUS.

\section{Summary}

There are multiple types of cystic lesions that can be found in the brain and in the posterior fossa. Among these, a new entity called MV PLUS (Multinodular and Vacuolating Posterior Fossa Lesions of Unknown Significance) has similar imaging characteristics to the vacuolating multinodular tumor, but as indicated by its acronym in English, it is located in the posterior fossa. These tumors are defined as a group of small, high intensity nodular subcortical images in the T2-FLAIR, with or without post-contrast enhancement. They can be differentiated from other cystic entities, because they are clustered lesions, without reactive inflammatory changes, in a cortex of normal appearance and without changes at follow-up. The latter, causes them to be considered benign, non-aggressive lesions. We present the case of a 21-year-old patient, who presented an incidentally found lesion in the central region of the vermis. MRI showed a multicystic-looking mass, that had no changes at follow-up in the last 3 years and without obvious differential diagnosis, suggestive of MV PLUS.

\section{Introducción}

El tumor multinodular vacuolante de fosa posterior (MVPLUS, por sus iniciales en inglés de Multinodular and vacuolating posterior fossa lesions of unknown significance) (1) es un nuevo tumor (2) que consiste en lesiones nodulares subcorticales agrupadas, con alta señal en secuencias T2-FLAIR, con o sin realce del medio de contraste, sin edema ni cambios inflamatorios y que se localiza en la fosa posterior $(1,2)$. Es un tipo de tumor neuronal vacuolante y nodular, pero, como la sigla lo indica, tiene localización en la fosa posterior

Recientemente, en un estudio retrospectivo de pacientes con sospecha de tumores neuronales multinodulares vacuolantes, Lecler y colaboradores encontraron que cuando esta entidad se ubica en la fosa posterior, en el $82 \%$ de los pacientes había una zona central de baja señal en el T2 que llamaron "el signo del punto central", que podría ser útil para la identificación de esta nueva forma de lesión (2).

A los pacientes que presentan esta entidad, se les ha realizado seguimiento mínimo de 24 meses sin que haya cambios imagenológicos, por lo que se considera que esta condición tiene un comportamiento benigno (1-3).

\section{Presentación del caso}

Se trata de un hombre de 21 años de edad, mestizo, con antecedente personal de hipogonadismo central y anosmia, a quien a sus 18 años se le practicó resonancia magnética nuclear (RMN) de silla turca por los antecedentes descritos, con diagnóstico de síndrome de Kallman: dismorfia de las circunvoluciones rectas, con surcos olfatorios marcadamente hipoplásicos. De manera incidental se encontró una lesión en el vermis. 
Al practicar RMN cerebral para visualizar la lesión en el vermis, se encontró en la región central una imagen de aspecto multiquístico conformada por lesiones milimétricas, multinodulares, redondeadas, con alta señal en secuencia T2-FLAIR (figuras 1 y 2), de baja señal en secuencias con información $\mathrm{T} 1$, sin edema, sin realce con el medio de contraste (figura 3), y sin efecto compresivo sobre el cuarto ventrículo. En la espectroscopia - Multivoxel CSI 3D (TE 35 y TE 135) y Single Voxel SVS con TE 30- realizada sobre la lesión no se identificaron metabolitos anormales. En particular no había elevación de la colina; tampoco presentaba restricción a la difusión ni efecto de susceptibilidad. Aunque se realizaron imágenes con información T1 y T2 de alta resolución no se identificó "el signo del punto central”. No se encontró compromiso supratentorial. Se recomendó seguimiento periódico y en los estudios posteriores la lesión ha permanecido estable, tanto en tamaño como en características, por los últimos 3 años.

\section{Discusión}

Los diagnósticos diferenciales de las lesiones quísticas en la fosa posterior son múltiples, entre ellos se destacan:

- Gangliocitoma cerebral displásico (Lhermitte-Duclos). Tiene patrón estriado con intensidades de señal alternantes, que corresponden a las folias engrosadas. Puede generar efecto de masa en el cuarto ventrículo y generalmente es una lesión dispersa y con compromiso del hemisferio cerebeloso, por lo que fue excluido como diagnóstico (4).

- DNET (por sus iniciales en inglés de Dysembryoplastic neuroepithelial tumors). Aunque puede tener un aspecto multiquístico, fue descartado, ya que generalmente es supratentorial e intracortical; ocasionalmente presenta algún grado de realce con el medio de contraste y puede tener calcificaciones hasta en el $20 \%$ de los casos (4).

- Quistes parasitarios. Generalmente son múltiples y con un compromiso difuso (5). La falta de evolución hacia otros estadios y la ausencia de cambios inflamatorios reactivos hace poco probable este diagnóstico en el caso presentado.

- Espacios de Virchow Robin prominentes. Típicamente exhiben una señal similar al líquido cefalorraquídeo en todas las secuencias, mientras que los MVNT no se suprimen en secuencia FLAIR con información T2 como ocurre en estos espacios perivasculares (1).

- Hamartomas. También llamados heterotopias neuronales nodulares, en RM típicamente exhiben la misma señal del parénquima normal - aunque ocasionalmente pueden aparecer con alta señal en secuencias con información T2-FLAIR-, pero tienen una localización común (6), lo que descarta que se trate de ello en el paciente de este caso.

- Astrocitoma cerebeloso. Es una lesión frecuente en este grupo etario, quística, cerebelosa, el borde del quiste puede o no realzar con el medio de contraste y ocasionalmente se encuentra asociado con un nódulo mural. Tiene localización excéntrica y compromete un hemisferio cerebeloso, lo que no concuerda con la imagen del paciente de este caso, por lo que se excluyó como diagnóstico (7).
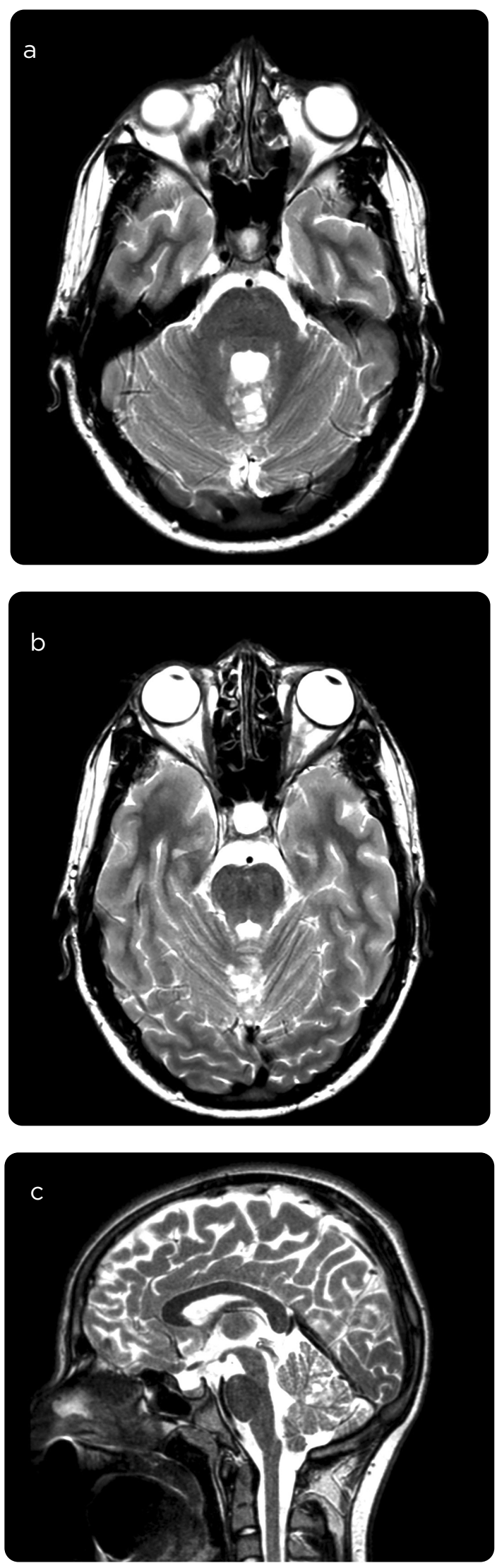

Figura 1. Imágenes multinodulares, coalescentes en la región central del vermis, de aspecto multiquístico, con alta intensidad de señal en secuencia TSE con información. T2 

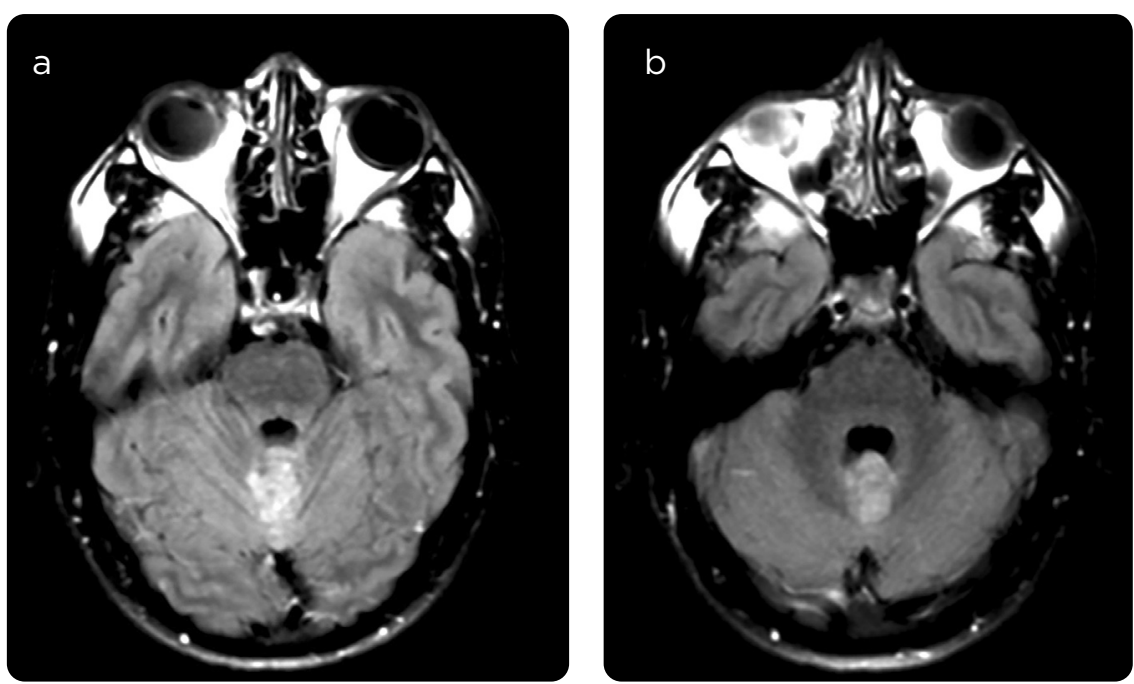

Figura 2. Imágenes multinodulares, coalescentes en la región central de aspecto multiquístico, con alta señal en secuencia T2-FLAIR.

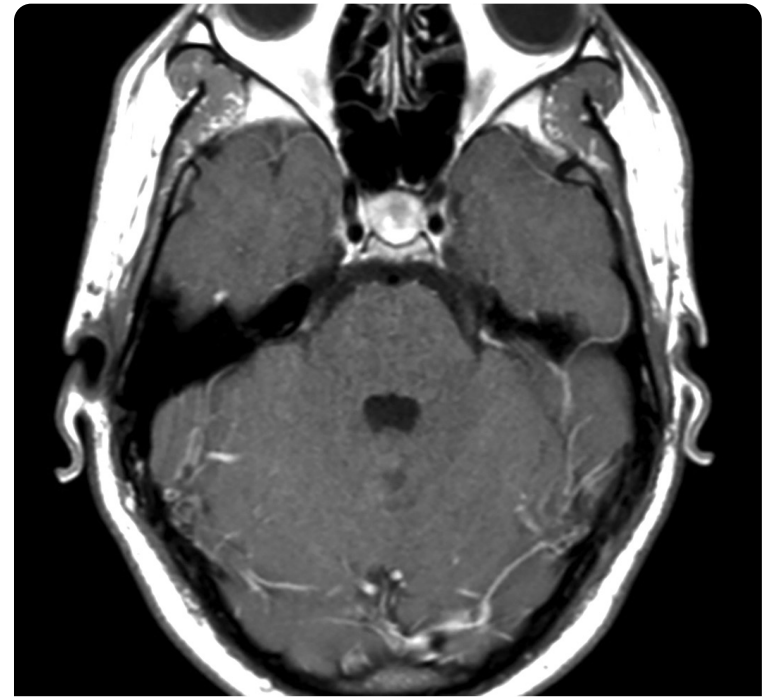

Figura 3. Secuencia TSE con información T1 con medio de contraste. La lesión multinodular en la región central del vermis no realza con la administración de medio de contraste.

En pacientes con distrofias musculares congénitas se pueden observar lesiones quísticas cerebelosas subcorticales; en estos casos las alteraciones están relacionadas con polimicrogiria y con un aspecto paquigírico variable, más en el área frontal. Estos pacientes presentan generalmente displasia cortical cerebelosa; ninguno de estos últimos se relacionan con las imágenes del paciente (8).

Una vez descartados los anteriores diagnósticos, se concluyó que la lesión visualizada en nuestro paciente corresponde muy probablemente a un MVPLUS.

Como se expuso, dentro de los diagnósticos diferenciales de masas quísticas en la fosa posterior debe incluirse el MVPLUS. Específicamente, en lesiones agrupadas nodulares, de alta señal en el T2-FLAIR, que no generan efecto de masa, sin realce con el medio de contraste, sin alteraciones en los metabolitos en la espectroscopia y que son invariables en el tiempo.
La descripción de más casos, el seguimiento de los mismos y la constante actualización de los especialistas en radiología, permite clasificar adecuadamente lesiones no tan comunes y conocer qué tipo de seguimiento e intervención requieren los pacientes afectados. Se debe continuar el estudio de este tipo de neoplasias y la descripción de un mayor número de series, ya que se considera que la falta de informes sobre esta entidad lleva a un subregistro en la literatura médica debido al sesgo de informe por falta de conocimiento y porque la mayoría de lesiones de este tipo probablemente no se resecan (1).

\section{Referencias}

1. Nunes RH, Hsu CC, da Rocha AJ, do Amaral LLF, Godoy LFS, Watkins TW, et al. Multinodular and vacuolating neuronal tumor of the cerebrum: A new "Leave Me Alone" lesion with a characteristic imaging pattern. Am J Neuroradiol. 2017;38(10):1899-904.

2. Lecler A, Bailleux J, Carsin B, Adle-Biassette H, Baloglu S, Bogey C, et al. Multinodular and vacuolating posterior fossa lesions of unknown significance. Am J Neuroradiol. 2019;40(10):1689-94.

3. Yamaguchi M, Komori T, Nakata Y, Yagishita A, Morino M, Isozaki E. Multinodular and vacuolating neuronal tumor affecting amygdala and hippocampus: A quasi-tumor? Multinodular and vacuolating neuronal tumor. Pathol Int. 2016;66(1):34-41.

4. Shin JH, Lee HK, Khang SK, Kim DW, Jeong AK, Ahn KJ, et al. Neuronal tumors of the central nervous system: Radiologic findings and pathologic correlation. RadioGraphics. 2002;22(5):1177-89.

5. Shih RY, Koeller KK. Bacterial, fungal, and parasitic infections of the central nervous system: Radiologic-pathologic correlation and historical perspectives: From the Radiologic Pathology Archives. RadioGraphics. 2015;35(4):1141-69.

6. Huse JT, Edgar M, Halliday J, Mikolaenko I, Lavi E, Rosenblum MK. Multinodular and vacuolating neuronal tumors of the cerebrum: 10 cases of a distinctive seizureassociated lesion: multinodular and vacuolating neuronal tumors. Brain Pathol 2013;23(5):515-24

7. Sutton LN. Cerebellar astrocytomas [internet]. Clinical Gate. 202AD. Disponible en: https://clinicalgate.com/cerebellar-astrocytomas/

8. Van der Knaap MS, Smit LME, Barth PG, Catsman-Berrevoets CE, Brouwer OF, Begeer JH, et al. Magnetic resonance imaging in classification of congenital muscular dystrophies with brain abnormalities. Ann Neurol. 1997;42(1):50-9.

\section{Correspondencia}

Valentina Múnera Orozco

Calle 31 \# 42A- 61 Ciudadela San Diego Núcleo

Medellín, Colombia

valentina.muneraorozco@gmail.com

Recibido para evaluación: 20 de abril de 2020

Aceptado para publicación: 21 de mayo de 2020 